http://jmscr.igmpublication.org/home/ ISSN (e)-2347-176x ISSN (p) 2455-0450

crossref DOI: https://dx.doi.org/10.18535/jmscr/v7i9.110

\title{
A Comparative Evaluation of Fosfomycin activity with other Antimicrobial agents against Enterobacteriaceae Uropathogen
}

\author{
Authors \\ Dr Ved Prakash Mamoria ${ }^{1}$, Kajal Meena $^{2}$, Dr Richa Sharma ${ }^{3 *}$ \\ ${ }^{1}$ Professor \& Head, Department of Microbiology, Mahatma Gandhi University of Medical Sciences \& \\ Technology, Jaipur \\ ${ }^{2}$ PGStudent, Department of Microbiology, Mahatma Gandhi University of Medical Sciences \& Technology, \\ Jaipur \\ ${ }^{3 *}$ Assistant Professor, Department of Microbiology, Mahatma Gandhi University of Medical Sciences \& \\ Technology, Jaipur \\ *Corresponding Author \\ Dr Richa Sharma
}

Assistant Professor, Department of Microbiology, Mahatma Gandhi University of Medical Sciences \& Technology, Jaipur, India

\begin{abstract}
Urinary Tract Infection is the common bacterial diseases that affect a large part of the world's population, in both hospitals and in the community. Escherichia coli, Klebsiella spp., and Proteus spp. are the uropathogens with the highest prevalence among patients with UTIs. The gender and sexual anatomy are among the major determinants of UTI. They are more common in women in comparison with men. In the present study, out of 591 Enterobacteriaceae isolates, majority of the urine specimens were from inpatients 377 (64\%) than from outpatients 214 (36\%). Out of 4176 urine specimens 591 (100\%) Enterobacteriaceae isolates were obtained, of which 432 (73.09\%) were Escherichia coli, followed by Klebsiella pneumoniae 97 (16.41\%), Providenciarettgeri21 (3.55\%), Proteus mirabilis 14 (2.37\%), Enterobacter cloacae 12 (2.03\%),Morganella morganii and Citrobacter koseri 6 (1.02\%), Serratia marcescens 3 (0.51\%). So total positive Enterobacteriaceae isolates were 591.Out of the 591 isolated patient's majority of isolates were from female patients in the age group of 21-30 i.e. 102 (17.26\%), followed by age group 31-40 i.e. 51(8.63\%). In the present findings, also reported the sensitivity to fosfomycin was significantly higher in E. coli i.e. 419(97\%), followed by Klebsiella pneumoniae 61 (62.9\%). It concludes that Fosfomycin may be given empirically in patients suffering from UTI due to Enterobacteriaceae.
\end{abstract}

Keywords: UTI, E.coli, Sensitivity, Uropathogens.

\section{Introduction}

Among the most common infectious diseases, urinary tract infections (UTIs), considered the most common bacterial diseases that affect a large part of the world's population, in both hospitals and in the community. It can be spread and caused by gram-negative bacteria such as Enterobacteriaceae particularly Escherichia coli, Klebsiella species, Enterobacter species, Citrobacter species and Proteus species ${ }^{(1)}$. E coli is the most common organism causing both community as well as hospital acquired UTI. The 
gender and sexual anatomy are among the major determinants of UTI. They are more common in women in comparison with men. UTI is rare in males unless microorganisms are introduced artificially with catheters. In women, the urethra is much shorter and very close to the anus, which is a constant source of faecal bacteria ${ }^{[2]}$. Escherichia coli, Klebsiella spp., and Proteus spp. are the uropathogens with the highest prevalence among patients with UTIs. However, the antibiotic susceptibility patterns of Enterobacteriaceae have been constantly changing due to the continuous development of new resistance mechanisms, like the production of extended-spectrum $\beta$-lactamases (ESBLs) or carbapenemases by bacteria and the spread of genes on mobile elements [3]. Fosfomycin is an old bactericidal antibiotic, discovered in Spain in 1969 from cultures of Streptomyces $^{[4]}$. That antibiotic have unique properties of not sharing any structural similarity and lack of cross-resistance with other antimicrobial agents. It inhibits cell wall formation by inhibiting the initial step involving phosphoenolpyruvate synthetase. Fosfomycin was previously used mainly as oral treatment for uncomplicated urinary tract infections (UTIs), currently attracts clinicians' interest worldwide. IDSA (Infectious Disease Society of America) and ESCMID (European Society of Clinical Microbiology and Infectious Diseases) recommends Fosfomycin as one of the first line agents for uncomplicated cystitis and pyelonephritis $^{[5]}$.

The emergence and spread of multidrug-resistant (MDR) Gram-negative bacteria related to urinary tract infections (UTIs) is increasing worldwide, both in hospitals and in the community. Fosfomycin tromethamine (FOF), a stable salt of Fosfomycin, has been found to be effective for the treatment of UTIs related to Escherichia coli, Citrobacter spp., Enterobacter spp., Klebsiella spp., Serratia spp., and Enterococcus faecalis ${ }^{[6]}$. The current study was undertaken to evaluate invitro activity of Fosfomycin against Enterobacteriaceae uropathogens and also assessed to compare Fosfomycin activity with the other antimicrobial agents against isolated Enterobacteriaceae uropathogens.

\section{Material and Methods}

The study was conducted prospectively in the Department of Microbiology at Mahatma Gandhi Medical College and Hospital Jaipur, Rajasthan, during one year period from June 2018 to May 2019 after receiving clearance from the Institutional Ethics Committee (IEC). The test group selected was the population of patients admitted in various OPD and IPD wards in the hospital regardless of their age, sex, occupation, religion and ethnicity.

\section{Source of Data}

Urine samples (4176) were received in lab between June 2018 to May 2019 for bacterial culture and sensitivity from various outdoor patient departments (OPDs) and indoor patient departments (IPDs) wards of Mahatma Gandhi Hospital (MGH) Sitapura Jaipur, Rajasthan.

\section{Collection of urine specimen}

Sample were collected with universal precautions by prescribed sterile techniques and transported to the laboratory as soon as possible maintaining optimum transportation condition. Detailed relevant history was taken as age, sex, the history of any in-dwelling medical devices used and the duration of wards and ICU stay. Urine samples were collected from various IPD and OPD wards.

(i) Mid-stream urine/ Urine in noncatheterise Patient: The clean catch midstream technique was employed to collect urine samples. Following the verbal consent of the patient /attendants, a midstream urine sample was collected in a wide mouthed sterile container with lid, labelled with the details of the patient. For clean catch mid-stream urine, patient will be instructed to cleanse the area with soaped swabs, then pass a small amount of urine 
into toilet, and finally urinate into the wide mouthed container.

(ii) Urine from Catheter: For catheterized patient-Urine was collected through the draining portal of the urinary catheter using aseptic precaution.

\section{Transport and storage of urine specimen}

After collection the urine sample, the container was properly labelled with patient's name, ID number etc. The specimens were then transferred to the laboratory as quickly as possible, usually within 1 hour after collection and processed as soon as possible. When the processing was delayed, they were stored at $4^{0} \mathrm{C}$.

\section{Processing of urine specimen}

Primary inoculation was done on Blood agar and Mac-conkey agar culture media using calibrated wire loop containing $0.001 \mathrm{ml}$ of urine sample. The inoculums were spread with the wire loop on the media plate. They were incubated aerobically at $37^{\circ} \mathrm{C}$ for $18-24$ hours. $>10^{5} \mathrm{CFU} / \mathrm{ml}$ for midstream urine $\&>10^{3} \mathrm{CFU} / \mathrm{ml}$ in catheterized urine sample was taken as significant Bacteriuria. Colony characteristics were noted of the bacterial growth. Then Gram's staining was done of the growth. Only gram-negative bacilli were further processed by battery of tests for identification of bacterial isolates. Only Enterobacteriaceae spp. of bacterial isolates were taken in this study. Then antimicrobial susceptibility testing was done by Kirby Bauer disc diffusion method and the interpretation of antibiotic susceptibility was interpret as per CLSI guidelines ${ }^{[7]}$. All culture media were obtained from Hi-media laboratories, Mumbai, India.

\section{Results}

The study was carried out in the Department of Microbiology during 12 months period from June 2018 to May 2019. A total of 4176 urine samples were studied from patients with clinically suspected cases of UTI. The result was analysed as follows; out of 4176 urine specimens 591
(100\%) Enterobacteriaceae isolates were obtained, of which $432 \quad(73.09 \%)$ were Escherichia coli, followed by Klebsiella pneumoniae 97 (16.41\%), Providencia rettgeri 21 (3.55\%), Proteus mirabilis 14 (2.37\%), Enterobacter cloacae 12 (2.03\%), Morganella morganii and Citrobacter koseri 6 (1.02\%), Serratia marcescens $3(0.51 \%)$. So total positive Enterobacteriaceae isolates were 591. Out of 591 (100\%) isolates of Enterobacteriaceae, majority were $E$. coli that is 432 (73.09\%), followed by Klebsiella pneumoniae 97 (16.41\%) (Figure 1). Of these 591 Enterobacteriaceae isolates,377 (63.79\%) were from IPD and 214 (36.21\%) were from OPD hospital wards as shown in Table 1.Of the 591 isolated patient's majority of isolates were from female patients in the age group of 21-30 i.e. 102 (17.26\%), followed by age group 31-40 i.e. 51(8.63\%) (Table 2, Figure 2). Sensitivity to fosfomycin was significantly higher in $E$. coli i.e. 419(97\%), followed by Klebsiella pneumoniae 61 (62.9\%) (Table 3, Figure 3).

Of the 591 urine Enterobacteriaceae isolates studied, out of 432 E. coli isolates highly sensitive to Tigecycline i.e. 427(98.9\%), followed by Colistin 424(98.1\%) and Fosfomycin 419(97\%).In total 97 Klebsiella pneumoniae isolates were highly sensitive to Colistin i.e. 94 (96.9\%), followed by Fosfomycin i.e. 61(62.9\%). In total 21 Providencia rettgeri isolates were highly sensitive to Fosfomycin i.e.15 (71.4\%), followed by Trimethoprim/ Sulfamethoxazole i.e. 4 (19.05\%). Out of 14 Proteus mirabilis isolates, 12 $(85.7 \%)$ isolates were sensitive to Fosfomycin and Piperacillin/Tazobactum, followed by 11 (78.6\%) Cefoperazone/Sulbactam, 10 (71.4\%) Ertapenem and Amikacin. In total 12 Enterobacter cloacae isolates were highly sensitive to Colistin i.e. 11 $(91.7 \%)$, followed by Tigecycline i.e. $8(66.7 \%)$ and $7(58.3 \%)$ Fosfomycin, Amikacin, Imipenem and Ertapenem. Out of 591 (100\%) Enterobacteriaceae isolates were highly sensitive to Colistin (90.5\%), Fosfomycin (89\%) and Tigecycline (82.7\%) (Table 4). 


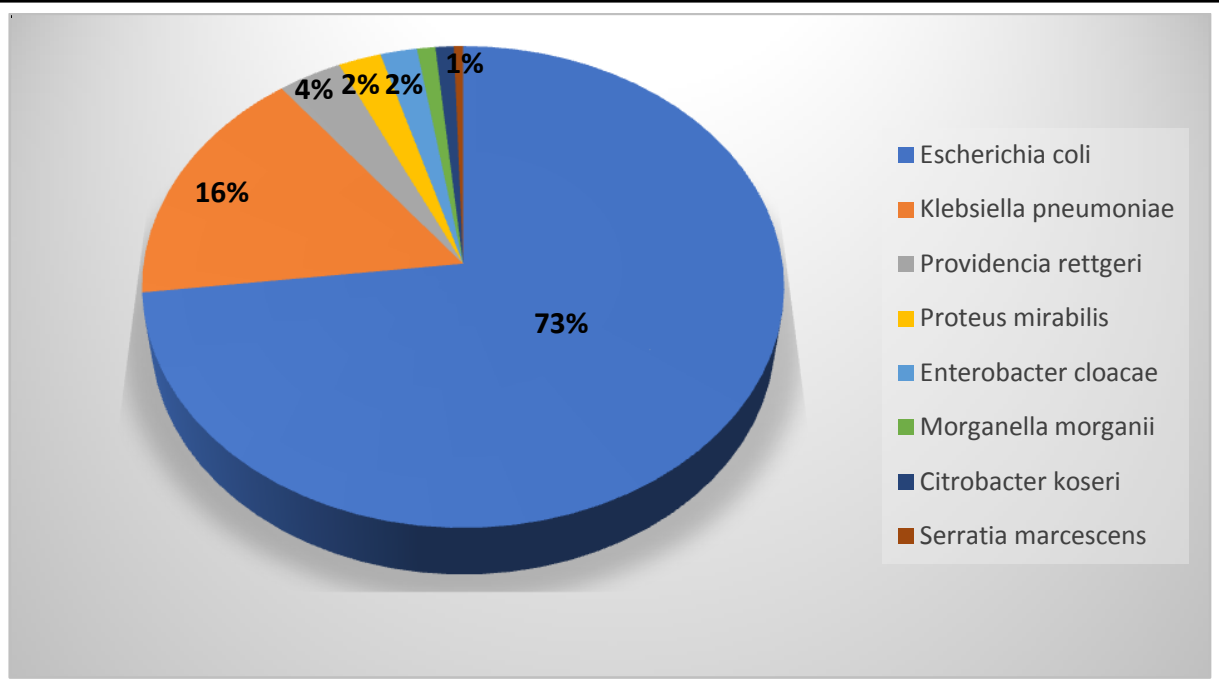

Fig. 1: Total No. of isolates obtained from Enterobacteriaceae family

Table 1: Distribution of positive Enterobacteriaceae isolated with respect to OPD/IPD

\begin{tabular}{|l|c|c|}
\hline Distribution of OPD/IPD & No. of isolates & Percentage \\
\hline OPD & 214 & $36.21 \%$ \\
\hline IPD & 377 & $63.79 \%$ \\
\hline Total & 591 & $100 \%$ \\
\hline
\end{tabular}

Table 2: Gender wise distribution of patients

\begin{tabular}{|l|c|c|c|}
\hline Age group & Male & Female & Total \\
\hline $0-10$ yrs. & 15 & 11 & 26 \\
\hline $11-20$ yrs. & 21 & 25 & 46 \\
\hline $21-30$ yrs. & 39 & 102 & 141 \\
\hline $31-40$ yrs. & 27 & 51 & 78 \\
\hline $41-50$ yrs. & 38 & 38 & 76 \\
\hline $51-60$ yrs. & 58 & 35 & 93 \\
\hline $61-70$ yrs. & 52 & 25 & 77 \\
\hline $71-90$ yrs. & 31 & 23 & 54 \\
\hline
\end{tabular}

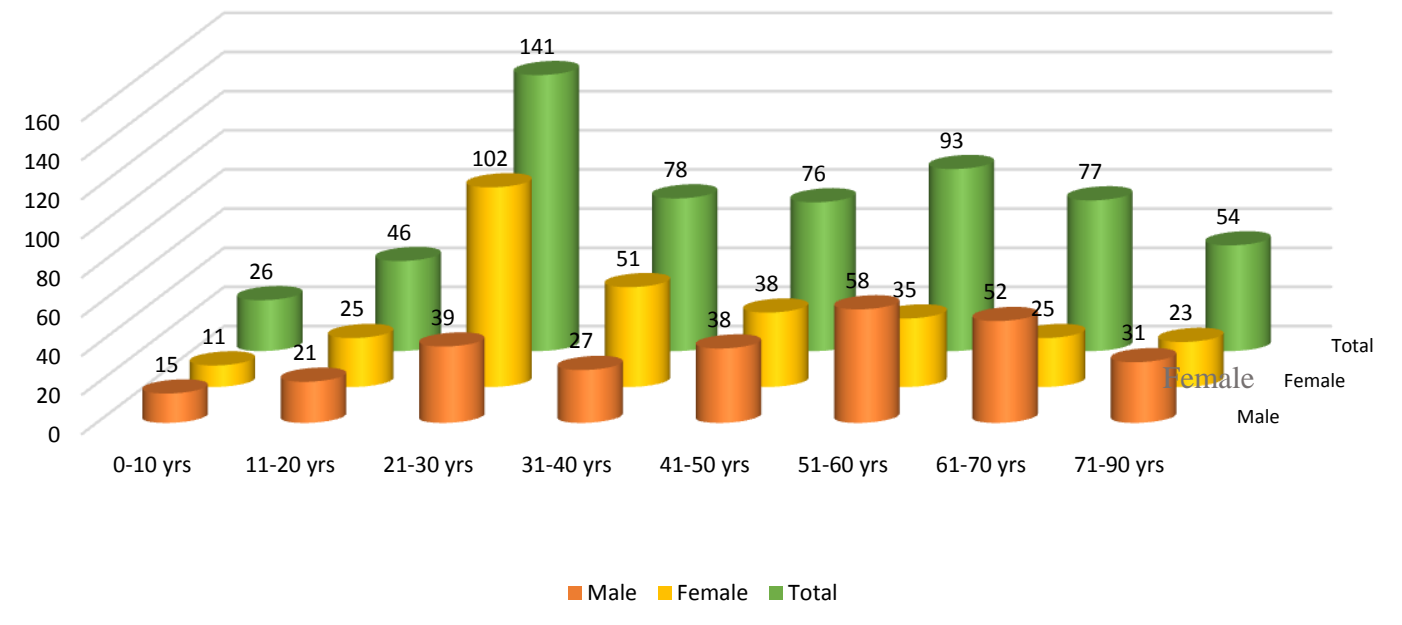

Fig. 2: Gender wise distribution of patients 


\section{JMSCR Vol||07||Issue||09||Page 634-640||September}

Table 3: Antibiotic susceptibility pattern of Enterobacteriaceae with respect to Fosfomycin

\begin{tabular}{|l|c|c|}
\hline \multirow{2}{*}{ Enterobacteriaceae spp. } & \multicolumn{2}{|c|}{ Fosfomycin susceptibility } \\
\cline { 2 - 3 } & S & R \\
\hline Escherichia coli & $419(97 \%)$ & $13(3 \%)$ \\
\hline Klebsiella pneumoniae & $61(62.9 \%)$ & $36(37.1 \%)$ \\
\hline Providenciarettgeri & $15(71.4 \%)$ & $6(28.6 \%)$ \\
\hline Proteus mirabilis & $12(85.7 \%)$ & $2(14.3 \%)$ \\
\hline Enterobacter cloacae & $7(58.3 \%)$ & $5(41.7 \%)$ \\
\hline Morganellamorganii & $3(50 \%)$ & $3(50 \%)$ \\
\hline Citrobacterkoseri & $6(100 \%)$ & - \\
\hline Serratia marcescens & $3(100 \%)$ & - \\
\hline Total & 526 & 65 \\
\hline
\end{tabular}

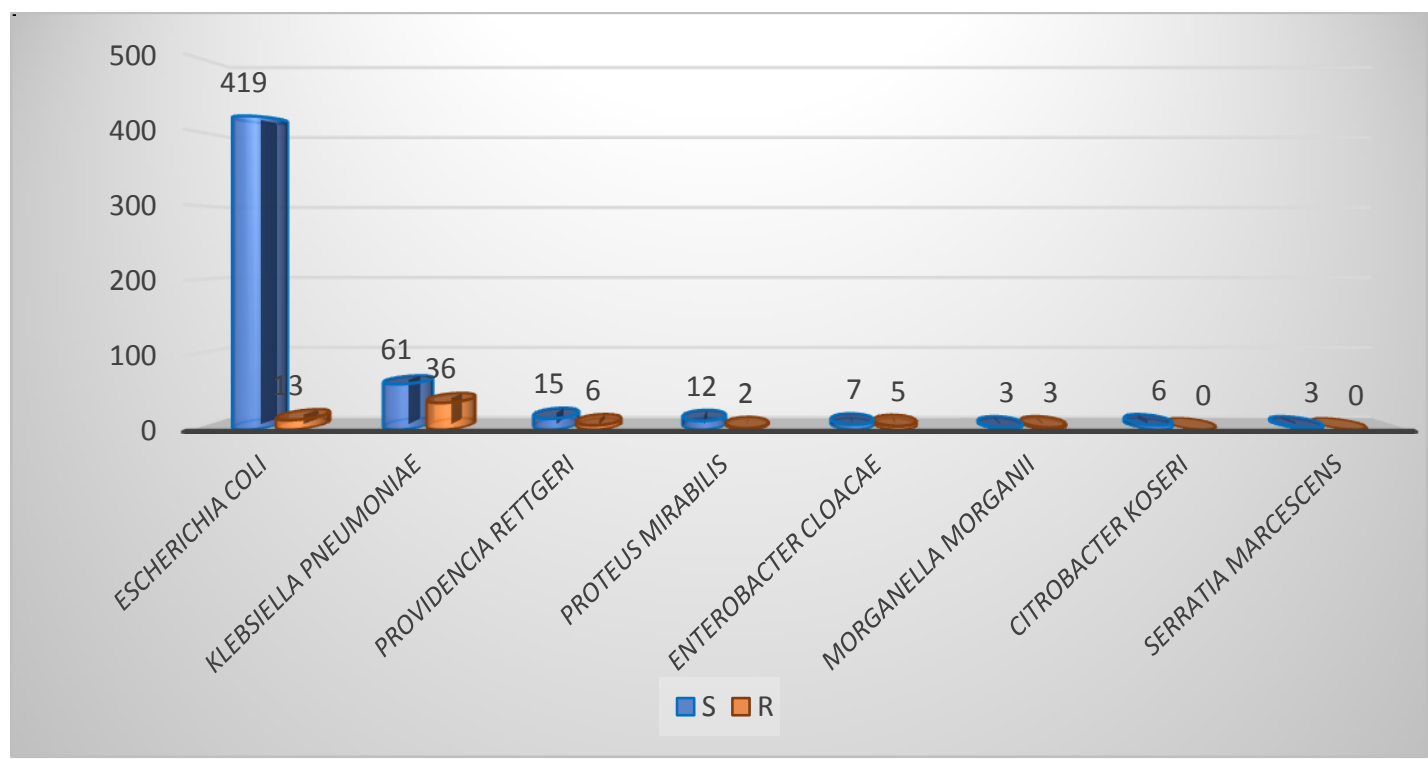

Fig. 3: Antibiotic susceptibility pattern of Enterobacteriaceae with respect to Fosfomycin

Table 4: Antibiotic susceptibility pattern of isolated Enterobacteriaceae spp.

\begin{tabular}{|c|c|c|c|c|c|c|c|c|c|c|c|c|c|c|c|c|c|c|}
\hline Entero. Spp. & $\begin{array}{l}\text { TOTA } \\
\text { L NO. }\end{array}$ & $\begin{array}{c}\mathrm{AM} \\
\mathrm{C}\end{array}$ & PIT & $\begin{array}{l}\text { CX } \\
\mathrm{M}\end{array}$ & CTR & $\mathrm{CPZ} / \mathrm{S}$ & CPM & ETP & IPM & $\mathrm{AK}$ & GEN & LE & $\begin{array}{c}\text { MIN } \\
\text { O }\end{array}$ & TGC & $\mathrm{FO}$ & NIT & $\mathrm{CL}$ & $\mathrm{COT}$ \\
\hline $\begin{array}{l}\text { Escherichia } \\
\text { coli }\end{array}$ & 432 & 128 & 234 & 66 & 78 & 249 & 121 & 285 & 303 & 324 & 227 & 82 & 239 & 427 & 419 & 313 & 424 & 145 \\
\hline $\begin{array}{l}\text { Klebsiella } \\
\text { pneumoniae }\end{array}$ & 97 & 28 & 36 & 19 & 24 & 38 & 28 & 40 & 42 & 52 & 43 & 28 & 30 & 46 & 61 & 26 & 94 & 39 \\
\hline $\begin{array}{l}\text { Providenciare } \\
\text { ttgeri }\end{array}$ & 21 & - & 1 & - & 1 & 1 & 2 & 1 & 1 & 1 & 2 & 1 & 2 & - & 15 & - & - & 4 \\
\hline $\begin{array}{l}\text { Proteus } \\
\text { mirabilis }\end{array}$ & 14 & 9 & 12 & 6 & 6 & 11 & 8 & 10 & 1 & 10 & 6 & 5 & 1 & - & 12 & - & - & 3 \\
\hline $\begin{array}{l}\text { Enterobacter } \\
\text { cloacae }\end{array}$ & 12 & - & 5 & - & 4 & 4 & 4 & 7 & 7 & 7 & 3 & 5 & 3 & 8 & 7 & 3 & 11 & 5 \\
\hline $\begin{array}{l}\text { Morganellam } \\
\text { organii }\end{array}$ & 6 & - & 5 & - & 3 & 5 & 4 & 4 & 2 & 5 & 3 & 1 & - & - & 3 & - & - & 1 \\
\hline $\begin{array}{l}\text { Citrobacterko } \\
\text { seri }\end{array}$ & 6 & 6 & 6 & 5 & 5 & 6 & 6 & 6 & 6 & 6 & 6 & 6 & 6 & 6 & 6 & 5 & 6 & 6 \\
\hline $\begin{array}{l}\text { Serratia } \\
\text { marcescens }\end{array}$ & 3 & - & - & - & - & 2 & 3 & - & - & 3 & 3 & 2 & 1 & 2 & 3 & - & - & 3 \\
\hline Total & 591 & 171 & 299 & 96 & 121 & 316 & 176 & 353 & 362 & 408 & 293 & 130 & 282 & 489 & 526 & 347 & 535 & 206 \\
\hline$\%$ & $100 \%$ & 29.1 & 50.6 & 16.2 & 20.5 & 53.5 & 29.8 & 59.7 & 61.3 & 69 & 49.6 & 21.9 & 47.7 & 82.7 & 89 & 58.7 & 90.5 & 34.9 \\
\hline
\end{tabular}

\section{Discussion}

This study shows the comparative evaluation of Fosfomycin activity with other Antimicrobial agents against Enterobacteriaceae Uropathogen in the Microbiology Department of Mahatma Gandhi
Medical College \& Hospital, Jaipur. Despite the widespread availability of antibiotics, Urinary Tract Infection (UTI) remains the most common bacterial infection in the human population. Antibiotic resistance is a common phenomenon in 
developing countries where drugs are available freely without prescription. The resistance pattern varies from one country to another. In the present study samples were obtained from various outdoor patient departments (OPDs) and indoor patient departments (IPDs) wards of Mahatma Gandhi Hospital (MGH) Sitapura Jaipur, Rajasthan.

In our study Out of 591 Enterobacteriaceae isolates, majority of the urine specimens were from inpatients 377 (64\%) than from outpatients 214 (36\%), which are in correlation with the findings of Ekadashi Rajni Sabharwal et al. ${ }^{[8]}$, reported $199(75.1 \%)$ urine specimens were from inpatients and 66 (24.9\%) were from outpatients. Similarly, to the study Sujatha R et al. ${ }^{[9]}$, reported $314(68.86 \%)$ urine specimens were from inpatients and 142 (31.14\%) were from outpatients.In our study highest prevalence was observed in female patients i.e. $310(52.45 \%)$ than from male patients $281(47.55 \%)$, and in the study of Gamal A. et al. ${ }^{[10]}$, reported highest prevalence in female patients $44(66.7 \%)$ than from male patients $22(33.3 \%)$ and study conducted by Thana Khawcharoenporn et al. ${ }^{[11]}$, reported $81 \%$ female and $19 \%$ male patient. The highest prevalence of UTI were observe in female patients rather than male patients because the female urethra is of particular importance to the pathogenesis of UTIs. The female urethra is relatively short compared with the male urethra and also lies in close proximity to warm, moist, perirectal region which is abundant with microorganisms. Because of the shorter urethra, bacteria can reach the bladder more easily in the female host.In present study, $591(100 \%)$ isolates from urine samples shows the Enterobacteriaceae growth, of which 432 (73.09\%) were Escherichia coli followed by 97 (16.41\%) Klebsiella pneumoniae, 21 (3.55\%) Providencia rettgeri, 14 (2.37\%) Proteus mirabilis, 12 (2.03\%) Enterobacter cloacae, 6 (1.02\%) Morganella morganii and Citrobacter koseri and $3(0.51 \%)$ Serratia marcescens, that is similar to the study conducted by Ekadashi Rajni Sabharwal et al. ${ }^{[8]}$, reported $68.8 \%$ Escherichia coli, followed by $24.9 \%$ Klebsiella spp. and
$5.28 \%$ Proteus spp. Similarly, to the study of Sujatha R et al. ${ }^{[9]}$, showed $260 \quad(57.02 \%)$ Escherichia coli, followed by Klebsiella 122 (26.75\%), Proteus 41 (8.99\%), Enterobacter 18 (3.95\%), Citrobacter 15 (3.29\%).

On studying the antibiotic susceptibility pattern for Fosfomycin, we found that 526 (89\%) is susceptible for Enterobacteriaceae isolates. In other similar studies given by Dr. Nandita pal et al. ${ }^{[12]}$, showed Fosfomycin was sensitive to 362 (93.29\%) Enterobacteriaceae isolates and Ekadashi Rajni Sabharwal et al. ${ }^{[8]}$, reported 249 (93.96\%) Fosfomycin susceptible to Enterobacteriaceae isolates. In another study of Sayantan Banerjee et al. ${ }^{[13]}$, overall 279 (97.21\%) Enterobacteriaceae isolates were susceptible to Fosfomycin, Similarly, to the study conducted by Asfia sultan et al. ${ }^{[14]}$, showed 368 (98.92\%) Fosfomycin sensitive Enterobacteriaceae isolates. It concludes that Fosfomycin may be given empirically in patients suffering from UTI due to Enterobacteriaceae. In our study, we observe that Colistin was sensitive to 535 (90.5\%) Enterobacteriaceae isolates. In other similar studies conducted by Dr. Nandita pal et al. (2017) [12] and Sayantan Banerjee et al. (2017) ${ }^{[13]}$, reported $373(96.13 \%)$ and $202 \quad(70.38 \%)$ Enterobacteriaceae isolates susceptible to Colistin respectively. In the present study, 408 (69.04\%) Enterobacteriaceae isolates were sensitive to Amikacin. Similarly, to the study conducted by Asfia sultan et al (2015) ${ }^{[14]}$ shows $362(97.31 \%)$ Enterobacteriaceae isolates were susceptible to Amikacin, Sayantan Banerjee et al. (2017) ${ }^{[13]}$ reported $232(80.84 \%)$ Enterobacteriaceae isolates were susceptible to Amikacin, Dr. Nandita pal et al. (2017) ${ }^{[12]}$ observe $332(85.57 \%)$ Enterobacteriaceae isolates were susceptible to Amikacin and Ekadashi Rajni Sabharwal et al. ${ }^{[8]}$, shows 162 (61.13\%) Enterobacteriaceae isolates were sensitive to Amikacin.

\section{Conclusion}

The present findings concluded that Colistin was most sensitive drugs and Fosfomycin is also 
comparably sensitive against Enterobacteriaceae isolates. But as Fosfomycin is cheaper in comparison to Colistin and it can be taken orally so for patients suffering from UTI caused by Enterobacteriaceae, Fosfomycin is a better option.

\section{Bibliography}

1. Beyene $\mathrm{G}$ and Tsegaye $\mathrm{W}$, Bacterial Uropathogens in Urinary Tract Infection and Antibiotic Susceptibility Pattern in Jimma University Specialized Hospital, Southwest Ethiopia. Ethiop. J. Health Sci. 2011 Jul;21(2):141-146.

2. Mollick S, Dasgupta T, Hasnain Md. J, Ahmed M. Isolation and Characterization of Pathogens Responsible for Urinary Tract Infection in Bangladesh and Determination of their Antibiotic Susceptibility Pattern. / Journal of Applied Pharmaceutical Science 6 (04); 2016: 072-076.

3. Tansarli GS, Athanasiou S, Falagas ME, Antimicrobial susceptibility of Enterobacteriaceae causing urinary 2 tract infections in Africa: Evaluation of the evidence. Antimicrob Agents Chemother, 2013;57(8): 3628-3639.

4. Falagas ME, Vouloumanou EK, Samonis G, Vardakas KZ, Fosfomycin. Clinical microbiology Reviews. 2016;29(2):321-347.

5. Patel B, Patel K, Shetty A, Soman R, Rodrigues C, Fosfomycin Susceptibility in Urinary Tract Enterobacteriaceae. J Assoc Physicians India. 2017 Sep;65(9):14-16.

6. Demir T, Buyukguclu T, Evaluation of the in vitro activity of fosfomycin tromethamine against Gram-negative bacterial strains recovered from community- and hospitalacquired urinary tract infections in Turkey. International Journal of Infectious Diseases. 17 (2013) e966-e970.

7. Clinical Laboratory and Standards Institute. Performance standards for antimicrobial susceptibility

testing

Twenty

first informational supplement M100: 2011;31(1).

8. Sabharwal ER, Sharma R, Fosfomycin: An Alternative Therapy for the Treatment of UTI Amidst Escalating Antimicrobial Resistance. JCDR, 2015; 9(12):DC06DC09.

9. Sujatha R and Pal N, Antibiotic resistance of the hospital and community acquired isolates of uropathogens in a teritiary care centre at Kanpur. Rama Univ. J. Med Sci, 2015; 1(1):10-17.

10. Gamal A. A-Ameri, Mawhoob N.O. Alkadasi, A.M.H. Sallam, Abdulrahman S. Naji and Abdulbaset A. zaid urinary tract infection of patients and antibiotic susceptibility patterns of Enterobacteriaceae in IBB city Yemen. Inter $\mathbf{J}$ Current Microbiology Applied Sci, 2014; 3(7): pp. 984-994.

11. Khawcharoenporn T, Vasoo S and Singh K, Urinary tract infections due to multidrugresistant Enterobacteriaceae: prevalence and risk factors in a Chicago emergency department; 2013; 10.1155.

12. Pal N, Majhi B, Evaluation of the spectrum of uropathogens, prevalent antimicrobial resistance and prospects of the newbie "Fosfomycin". IOSR-JDMS; 2017:16(9):PP 54-59.

13. Banerjee S, Sengupta M, Sarker TK, Fosfomycin susceptibility among multidrugresistant, extended-spectrum betalactamase-producing, carbapenem-resistant uropathogens. 2017; 33(2):149-154.

14. Sultan A, Rizvi M, Khan F, Sami H, Shukla I, Khan HM, Increasing antimicrobial resistance among uropathogens: is fosfomycin the answer? Urol Ann, 2015; 7(1):26-30. 